\title{
Evolution and potential function of fibrinogen-like domains across twelve Drosophila species Sumit Middha ${ }^{1,2}$ and Xinguo Wang*1
}

Address: ${ }^{1}$ Center for Genomics and Bioinformatics, Indiana University, Bloomington, IN 47405, USA and ${ }^{2}$ Bioinformatics Core, Mayo Clinic, Rochester, MN 55905, USA

Email: Sumit Middha - middha.sumit@mayo.edu; Xinguo Wang* - xinguowang0@gmail.com

* Corresponding author

Published: 30 May 2008

BMC Genomics 2008, 9:260

This article is available from: http://www.biomedcentral.com/I47I-2/64/9/260

(c) 2008 Middha and Wang; licensee BioMed Central Ltd.

This is an Open Access article distributed under the terms of the Creative Commons Attribution License (http://creativecommons.org/licenses/by/2.0), which permits unrestricted use, distribution, and reproduction in any medium, provided the original work is properly cited.
Received: 30 November 2007

Accepted: 30 May 2008

\begin{abstract}
Background: The fibrinogen-like (FBG) domain consists of approximately 200 amino acid residues, which has high sequence similarity to the $\mathrm{C}$-terminal halves of fibrinogen $\beta$ and $\gamma$ chains. Fibrinogen-related proteins (FREPs) containing one or more FBG domains are found universally in vertebrates and invertebrates. In invertebrates, FREPs are involved in immune responses and other aspects of physiology. To understand the complexity of this gene family in Drosophila, we analyzed FREPs in twelve Drosophila species.

Results: Using the genome data from 12 Drosophila species, we identified FBG domains in each species. The results show that the gene numbers in each species vary from 14 genes up to 43 genes. Using sequence profile analysis, we found that FBG domains have high sequence similarity and are highly conserved throughout. By comparison of structure and sequence conservation, some of the FBG domains in Drosophila melanogaster are predicted to function in recognition of carbohydrates and their derivatives on the surface of microorganisms in innate immunity.

Conclusion: Sequence and structural analyses show that FREP family across 12 Drosophila species contains conserved FBG domains. Expansion of the FREP families in Drosophila is mainly accounted by a major expansion of FBG domains.
\end{abstract}

\section{Background}

In mammals, fibrinogen is composed of six polypeptide chains, two each of the $A \alpha, B \beta$ and $\gamma$ chains. Fibrinogen is a soluble plasma protein, which participates in both the cellular phase and the fluid phase of coagulation [1]. The fibrinogen-like (FBG) domain consists of approximately 200 amino acid (aa) residues and has high sequence similarity to the C-terminal halves of fibrinogen $\beta$ and $\gamma$ chains. However, the two loops in the fibrinogen $\gamma$ fragment are shortened by 14 and 7 aa in FBG domain respectively $[2,3]$. Fibrinogen-related proteins (FREPs) contain one or more FBG domains, which are found universally in vertebrates and invertebrates [2,3]. In mammals, three distinct fibrinogen-related proteins have been identified: ficolin, tenascins, and microfibril-associated protein (MAP) [4-6]. All of these FREPs contain an FBG domain in their $\mathrm{C}$ terminus, but differ in their $\mathrm{N}$-terminal regions. Ficolins are the most important molecules that have been studied so far, and they act as pattern recognition receptors to bind pathogens in host innate immunity initiating immune responses [4,7-9]. The FBG domain in ficolins is able to form polymer through collagen O-like triple helices, and is responsible for $\mathrm{N}$-acetylglucosamine (GlcNAc) and other sugar binding activity [4,7-9]. Recent studies 
have shown that human serum ficolins act as phagocytic receptors on circulating monocytes for microorganism recognition [10].

In invertebrates, several FREPs have been reported in various species, such as tachylectins from the horseshoe crab, Tachypleus tridentatus [2], fibrinogen-related proteins (FREP) from the snail, Biomphalaria glabrata [11], ficolins from the solitary ascidian, Halocynthia roretzi [12], tachylectin-related protein in the sponge, Suerites domuncula [13] and aslectin from the mosquito, Armigeres subalbatus [14]. All of these FREPs contain a common C-terminal FBG domain. These FREPs likely play an important role in the innate immune response against parasites $[2,13,14]$. The FBG domain of tachylectin is able to bind GlcNAc [2]. Aslectin can bind GlcNAc and bacteria, and therefore it is likely involved in the antibacterial immune response in mosquitoes [14]. Recently we characterized the FBG domains of FREPs genome-wide in mosquito, Anopheles gambiae, and predicted that some of the FBG domains may function by binding to pathogens in host immune response [3]. The FBG domain proteins have been identified and characterized recently with respect to immune response in mosquito [15].

Comparative genome analysis of related species provided a powerful and general approach for identifying functional elements without previous knowledge of function $[16,17]$. It has substantial power to identify genes, define gene structure, highlight rapid and slow evolutionary change, identify regulatory elements and reveal combinatorial control of gene regulation. Furthermore, it allows identification of all the major differences among the organisms [16-18]. The power is comparable to experimental analysis in terms of sensitivity and precision [17]. With the 11 additional Drosophila species genomes sequenced, this extensive sequence resource, encompassing species with well-defined phylogenetic relationships, provides a model system for comparative genomic analyses [19]. In this study, we apply sequence profile analysis and comparative genomics to the wealth of new information from 12 Drosophila species genomes to identify FBG domains in FREPs. Provided is an overview of FREP gene family, including sequence alignments, patterns of conservation, phylogenetic relationships and potential function.

\section{Results and Discussion}

Fibrinogen-related proteins and fibrinogen-like domain in the 12 Drosophila species

Fibrinogen-like domains have been well defined in mammals and insects $[2,3,20]$. In order to select FBG domain seed in Drosophila melanogaster, human ficolins were used to BLAST against D. melanogaster proteins. The resulting sequences were aligned and the FBG domain of
NP_611160 (FBgn0034160) was selected as a seed to perform a BLAST search. Meanwhile, sixty amino acids were used as the minimum length of homology, and protein sequences having $35 \%$ or greater amino acid identity were chosen as FREP protein. This search identified 285 FREP proteins in the genome of 12 Drosophila species (Table 1). The data showed that the numbers of FREPs in each Drosophila species vary from 14 genes up to 43 genes. All the species in Drosophila subgenus have more than 20 genes while four of the eight species in Sophophora subgenus have more than 20 genes (Table 1). D. Yakuba has the least gene number with just 14 genes, and $D$. grimshawi has most gene number, with 43 FREP genes, followed by $D$. willistoni and D. virilis with 34 and 33 FREPs respectively (Table 1). Unlike olfactory receptors for which the gene number in each species has been quite stable during evolution [21], the numbers of FREP genes are divergent in Drosophila species, suggesting this gene family evolved under relaxed constraints.

To detect known domain structure in the FREP proteins, the SledgeHMMER program was used to scan the protein sequences $[22,23]$. The data showed that all the FREP proteins contain at least one FBG domain (Additional file 1). Interestingly, four FREP proteins contain multiple FBG domains, e.g. dgri _7049, dgri_7054 and dana _11471 contain 2 FBG domains and dwil_15059 has 3 FBG domains (Additional file 1). Most of the FREP proteins have one FBG domain located in the C-terminus. In these FREP proteins, we found that the majority contain a full length FBG domain composed of approximately 200 amino acids, which is similar to the full length FBG domains in human and mosquitoes $[2,3,20]$. However, some of the FREPs contain a truncated FBG domain (Table 1 and Additional file 1). This could be caused by sequencing error or automated annotation $[3,22]$. Besides FBG domains in FREP proteins, we also found that 14 of FREP proteins contain other known domains; detailed information is available in Additional file 1.

\section{Phylogenetic relationship of fibrinogen-like domains and chromosomal location of fibrinogen-related proteins}

To analyze the evolutionary history of FBG domains in the FREP family, a phylogenetic tree was constructed with the alignments of the conserved FBG domains using Neighbor Joining. The striking pattern in this tree is that of FBG domains from different species being grouped together, indicating that they are orthologous genes (Fig. 1A, Table 2 and Additional file 2). This suggests that these FBG domains are stable across Drosophila species during evolution. We also found that some clusters are composed of FBG domains only from one species, such as $D$. grimshawi (Fig. 1B and Table 2), suggesting that gene expansion occurred in D. grimshawi after divergence of this species from the others. The FBG domains from D. willistoni 
Table I: The gene number of fibrinogen-related proteins in 12 Drosophila species

\begin{tabular}{llcc}
\hline Subgenus & Species & Gene Number & Truncated FBG \\
\hline \multirow{2}{*}{ Sophophora } & melanogaster & 17 & 1 \\
& simulans & 14 & 2 \\
& sechellia & 15 & 0 \\
& yakuba & 14 & 1 \\
& erecta & 16 & 1 \\
& ananassae & 28 & 2 \\
& pseudoobscura & 25 & 2 \\
& persimilis & 24 & 3 \\
& willistoni & 34 & 6 \\
Drosophila & virilis & 33 & 2 \\
& mojavensis & 22 & 3 \\
& grimshawi & 43 & 13 \\
\hline
\end{tabular}

formed two separate branches far apart in the tree (Additional file 2). Furthermore, some clusters are composed of FBG domains from a few Drosophila species instead of all, e.g, the majority of FBG domains from $D$. virilis, $D$. mojavensis and D. grimshawi are clustered together, and FBG domains from $D$. persimilis and D. pseudoobscura form a cluster as well (Fig. 1C, Table 2 and Additional file 2). Interestingly, in the evolutionary tree, for $D$. virilis, $D$. mojavensis and D. grimshawi of Drosophila subgenus, the FBG domains are clustered together and far apart from Sophophora subgenus, suggesting that the gene divergence had occurred after divergence of these three species from the others.

To understand the evolutionary history of this gene family across closely related species, we also compared the correlations between chromosomal locations of FREP and sequence similarities of FBG domains among the family members. Gene locations for the FREP family have been retrieved from the AAA database [24]. None of the FREP genes were observed on the dot chromosome, which is chromosome 4 in D. melanogaster. Some of FREP genes

Table 2: Numbers of fibrinogen-related proteins for 14 clades in each Drosophila species

\begin{tabular}{|c|c|c|c|c|c|c|c|c|c|c|c|c|c|c|}
\hline Speci & A & B & C & $D$ & $E$ & $\mathrm{~F}$ & G & $\mathrm{H}$ & I & J & K & L & $M$ & \\
\hline & 2 & I & r & I & 0 & 2 & I & 2 & 0 & 3 & 0 & 0 & & \\
\hline & 2 & I & 1 & 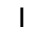 & & 1 & 0 & & & 2 & & & & \\
\hline & & I & I & & & 2 & & & & & & & & \\
\hline & & I & I & & & & & & & & & & & \\
\hline & & I & 1 & I & & 1 & I & & 0 & 3 & 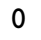 & I & & \\
\hline & & 2 & 0 & 1 & & 7 & 4 & & I & 2 & 0 & I & & \\
\hline & 2 & 0 & 2 & 1 & & 7 & 0 & & 0 & 3 & & I & & \\
\hline & 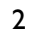 & 0 & I & I & & 8 & 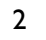 & & 0 & 4 & & I & & \\
\hline$w i$ & & I & 8 & I & & 4 & 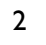 & I & I & 2 & & 0 & & \\
\hline virilis & 2 & 2 & 1 & I & & 1 & 6 & 3 & 0 & I & I & 0 & 1 & \\
\hline mojav & 2 & I & 0 & 1 & & I & 6 & 0 & 1 & 2 & I & 0 & I & 0 \\
\hline grimshawi & I & 2 & 0 & 1 & 3 & 0 & 8 & 2 & 0 & 2 & 7 & 0 & I & \\
\hline
\end{tabular}

are arrayed in tandem and form clusters (Fig. 2), especially the clusters in the Drosophila subgenus of D. mojavensis, D. virilis and D. grimshawi, and the similar pattern was also observed in Sophophora subgenus, including D. pseudoobscura, D. persimilis and D. wilistoni (Fig. 2). If the number of FBG domains increased mainly by tandem duplication, we would expect the domains which are physically clustered in the genome to form a monophyletic group. By examining the relationships between phyletic pattern and chromosomal location of the FBG domains, this pattern was only identified in D. wilistoni, which is that the genes clustered on a single $\mathrm{D}$. wilistoni scaffold occurred to be in close-by branch in the phylogenetic tree (Fig. 2 and Additional file 2). The vast majority of FBG domains closely located in the genome are scattered in different clusters in the phylogenetic tree (Fig. 2 and Additional file 2). This suggests that a dynamic history for the FBG domains likely involved shuffling among chromosomes. The predicted role, for at least a subset of these FBG domains, is in carbohydrate sensing (see below). This expansion in the Drosophila genome may have been a response to the diversity of carbohydrates encountered, resulting in the utilization of numerous FBG domain variations in order to recognize a broad range of different carbohydrates.

\section{Conserved structure of the FBG domains and their potential function}

In order to construct an optimal multiple alignment of the FBG domain, we first aligned selected sequences from $D$. melanogaster with the T_Coffee program $[25,26]$ (Fig. 3). Multiple alignment of the FBG domain sequences showed that FBG domains are highly similar throughout. Using the multiple alignments of the FBG domains as queries, the secondary structure was predicted with the PHD program [27], which showed that the FBG domains have a highly conserved structure profile (Fig. 3). To further compare the predicted secondary structure of the FBG domains with known structures, we found that the FBG domain is structurally related to the FBG domains of human ficolin and TL5A in the protein data bank (PDB) $[20,28]$. The FBG domains of human ficolin and TL5A comprise of a central and larger domain $\mathrm{B}$ and a relatively smaller domain P $[20,28]$. The domain $B$ is predominantly built up by a twisted seven stranded anti-parallel $\beta$ sheet (strands $\beta 3-\beta 7, \beta 9$ and $\beta 12$ ) and helices $\alpha 4$ and $\alpha 5$ functioning as the backbone. The domain $\mathrm{P}$ possesses only a few short elements of secondary structure, and comprises the major functional site forming a binding pocket $[20,24]$. The predicted secondary structures of the FBG domains in the FREP gene family approximately correspond to the domain architectures of FBG domains in human ficolin and TL5A (Fig. 3). The $\beta$-sheets and $\alpha$-helices in the predicted structure of the FBG domain are highly conserved with the corresponding structures in 


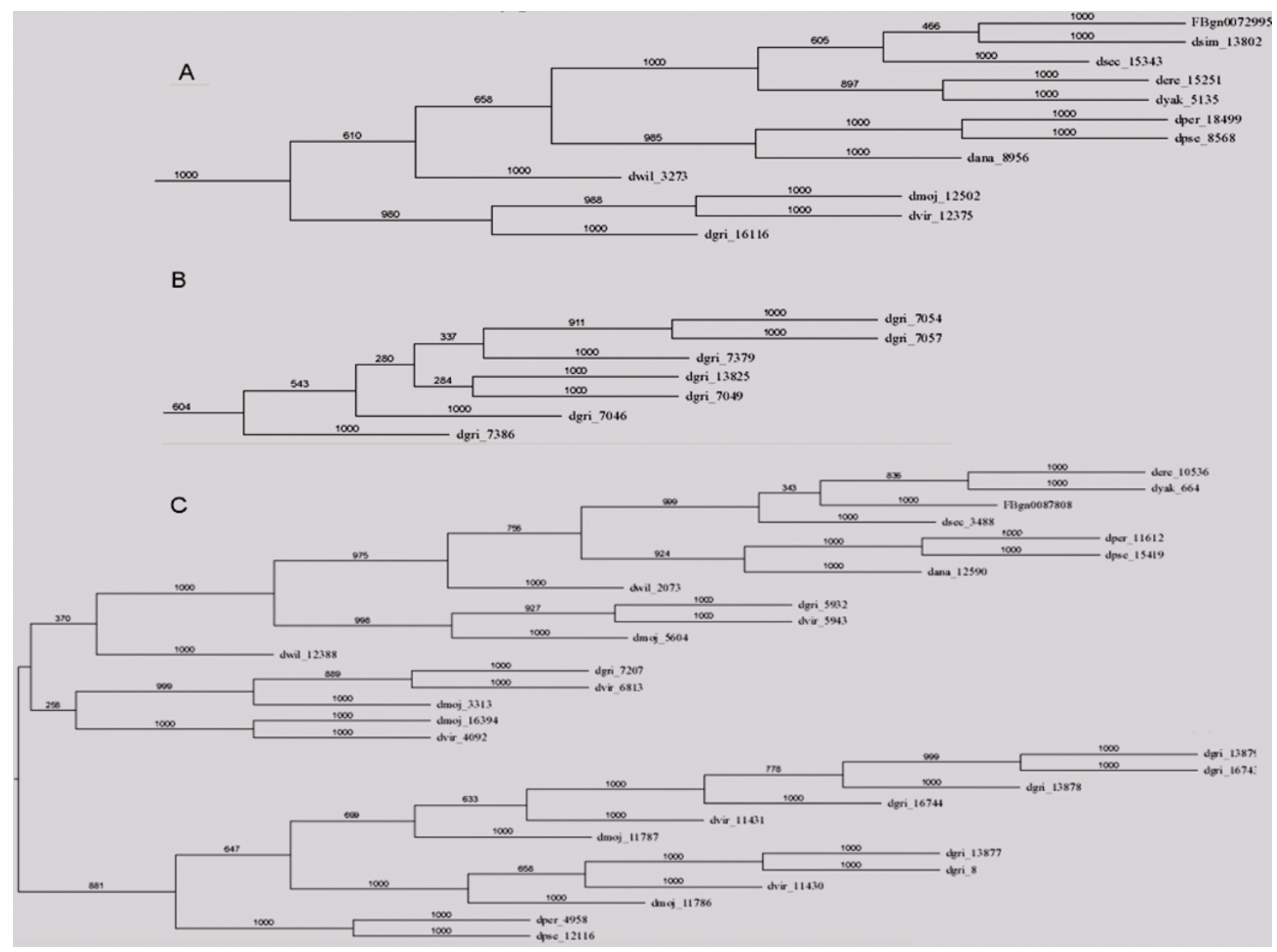

\section{Figure I}

Selected clusters of phylogenetic tree of the FBG domains in the 12 Drosophila species. The seed sequence used for constructing the tree was the multiple sequence alignment of FBG domains that excluded truncated FBG domains. Bootstrap was applied to the data. Protein distance was calculated using the Jones-Taylor-Thornton model of change between amino acids and a Hidden Markov Model (HMM) method of inferring different rates of evolution at different amino acid positions. Neighbor-joining was applied to produce the tree. The FBG domains of each FREP are denoted by their gene name or GLEANR gene ID. A. A cluster composed of FBG domains from multiple species (Group D in supplement fig. 2). B. A cluster composed of FBG domains from a single species (D. grmshawii, Group K in supplement fig. 2). C. Majority of FBG domains from D. virilis, D. mojavensis and D. grimshawi are clustered together (Group $G$ in supplement fig. 2).

TL5A, especially in the domain B (Fig. 3). For example, the central strand $\beta 12$, which extends the $C$ terminus of domain $P$ back to domain $\mathrm{B}$ and brings both polypeptide termini in close proximity, was also seen in Drosophila FBG domains (Fig. 3). This suggests that the FBG domain architecture is conserved among human, horseshoe crab and D. melanogaster. The projection of some of the highly conserved domains that form the ligand-binding pocket suggests that the core structure of the ligand-binding pocket is also likely to be conserved across these FBG domains (Fig. 3). These observations imply that the FBG domains are most likely to function as receptors for carbohydrates or their derivatives. Beyond the common core, FBG domains also show great diversity in terms of the insertions and deletions among the conserved domains, for example, NP_476710 loses a conserved domain due to deletion and NP_647820 has a short insertion located in the loop region (Fig. 3). By comparison of amino acids in the FBG domains of FREP corresponding to the P domain binding site in TL5A, we found that the domain architectures of these FBG domains have considerable diversity that is incorporated into a shared basic architectural blue- 
dana_scaffold_13266

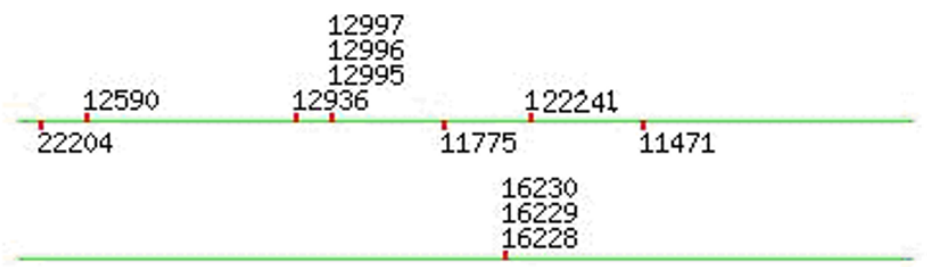

dana_scaffold_12916

dana_scaffold_12943

$\begin{array}{lll}709 & 747 & 746 \\ 7 & 779 \\ & 22434 \quad 532 \\ & 564 & 565\end{array}$

dere_scaffold_4845

dpse_Ch4_group1

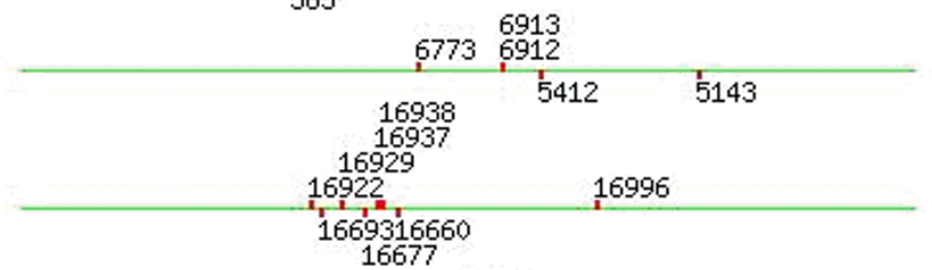

dper_scaffold_5

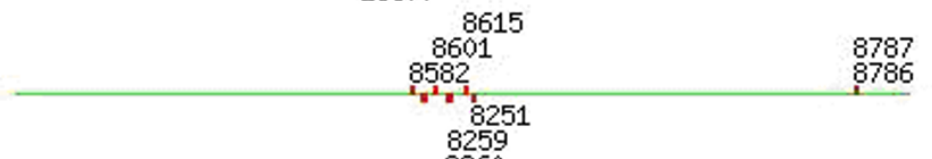

dwil_scaffold_180708

dmoj_scaffold_6496

dvir_scaffold_13049

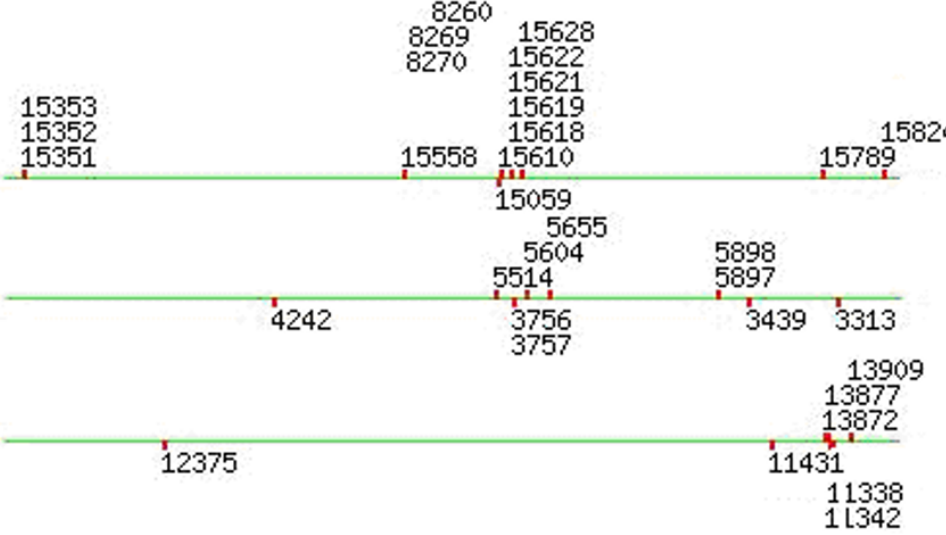

dvir_scaffold_12875
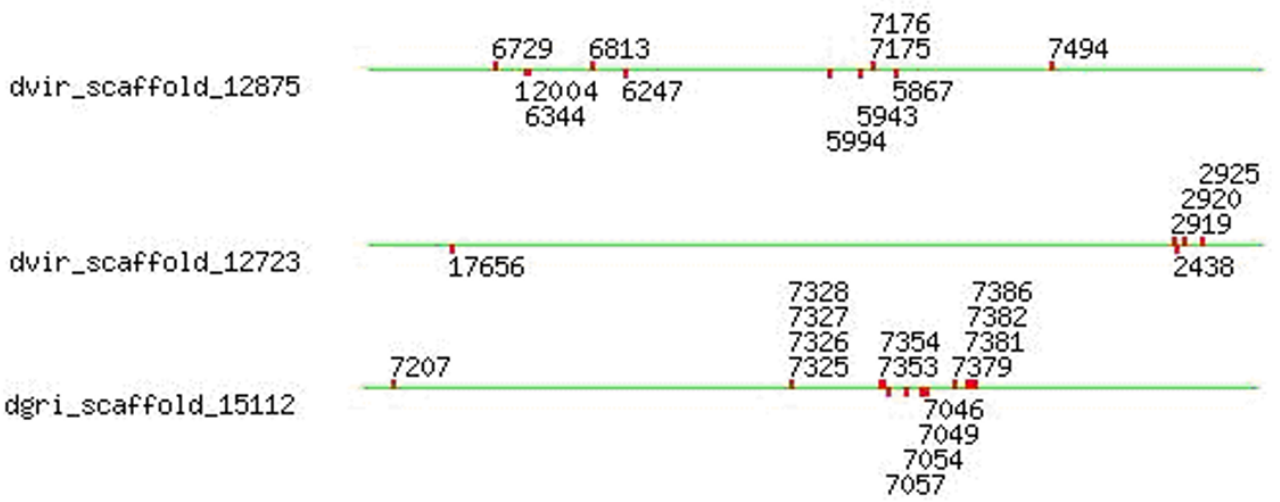

Figure 2

Scaffold location of selected FREPs in the Drosophila species. Gene locations for the FREP family were retrieved from the AAA database. Scaffold was named using single letter from genus plus the first three letters from subgenus to differentiate species. The scaffold is represented with a green line, which is not scaled. The relative location for each gene was shown on the scaffold using their GLEANR gene ID. 

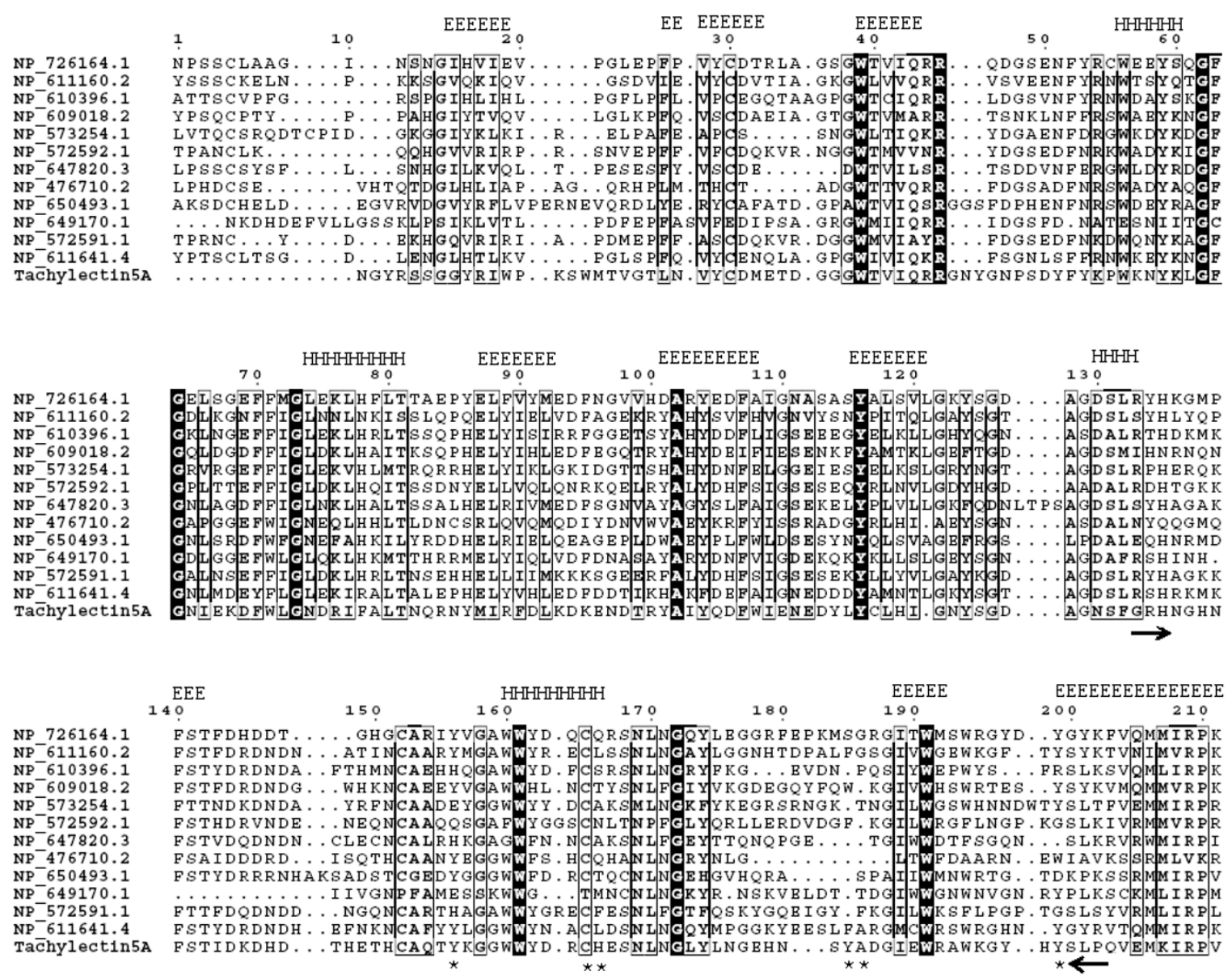

\section{Figure 3}

Multiple sequence alignment of a representative set of the FBG domains in D. melanoganster. Multiple sequence alignment was constructed using T-Coffee program. The 100\% consensus sequence was boxed with black in the alignment. The PHD secondary structure is shown above the alignment with $\mathrm{H}$ representing an $\alpha$-helix and $\mathrm{E}$ representing a $\beta$-strand. The sequences are denoted by their gene names in GenBank. The domain $\mathrm{P}$ is indicated between two arrows. The amino acids involved in forming binding pocket were shown in star.

print (Fig. 3). In invertebrates, several FREP proteins have been reported to play an important role in innate immunity and in particular in the recognition of parasites $[2,13,14]$. Aslectin can be upregulated by bacterial challenge and is able to bind GlcNAc and bacteria [14]. The FBG domain of TL5A can form a ligand-binding pocket specifically recognizing the acetyl-group in eliciting an immune response [20]. These data suggest that the FBG domains of FREPs in D. melanogaster probably function in recognizing carbohydrate moieties in innate immunity. These were further supported by microarray analysis in melanogaster from GEO profile [29]. NP_611160 was upregulated by virus infection, fugal infection and injury [30,31]. NP_649170 was upregulated in cell line following LPS or E. coli infection [31]. Phenobarbital is a xenobiotic that triggers a defense response, inducing genes that encode key detoxification enzymes. DHR96 is a xenobiotic receptor that controls metabolic and stress-response genes [32]. NP_573254 is down-regulated following Phenobarbital treatment, but is not affected by DHR96. However, NP_647820 and NP_723894 are regulated by DHR96 receptor [32]. These suggest that FREP genes play important roles in both innate immunity and physiology.

\section{Conclusion}

The data demonstrated that the number of FREP genes in each Drosophila species vary from 14 genes up to 43 genes. Some of the FBG domains from different species are grouped together in the phylogenetic tree, indicating that they are orthologous genes. This suggests that these FBG domains are stable across Drosophila species in evolutionary process. We also found that some clusters in the tree 
are composed of FBG domains only from one species or a few species instead of all 12 Drosophila species. By comparison of phylogenetic tree and chromosomal location of FREPs, we found that expansion of the FREP families in Drosophila is mainly accounted for by a major expansion of FBG domains, and both tandem duplication and shuffling are involved in gene expansion of FREPs. The results from sequence and structural analyses imply that FBG domains are highly similar across 12 Drosophila species. Some of the FBG domains in Drosophila melanogaster are predicted to function in recognition of carbohydrates and their derivatives on the surface of microorganisms in innate immunity.

\section{Methods}

\section{Database searching and sequence retrieving for} fibrinogen-related protein

Fibrinogen-like domain seed sequence was used to align with the Drosophila proteome sequences using the BLAST search. Flybase version 5.1 data was used for D. melanogaster while Comparative Annotation Freeze 1 (CAF1) data was used for the other 11 Drosophila species [33,34]. The 11 Drosophila species other than D. melanogaster are D. simulans, D. sechellia, D. yakuba, D. erecta, D. ananassae, D. pseudoobscura, D. persimilis, D. willistoni, D. mojavensis, D. virilis, and $D$. grimshawi. The genome sequences are available from the Assembly, Alignment and Annotation of the new 12 related Drosophila genomes [24]. The genus name will be omitted in results and discussion. Translation sequences of GLEANR gene models we used for the 11 Drosophila species were downloaded from AAA datasets on January 2007. The resulting protein sequences from the first search were used to iterate the search and retrieve any left out FREPs. Sixty amino acids was used as a minimum length of the match along with 35\% homology in order to add a protein to the list of FREPs. The list was manually checked and a non-redundant set of protein sequences was obtained.

\section{Domain identification in Drosophila fibrinogen-related proteins}

SledgeHMMER was used to carry out batch searching of the current Pfam database (version 20.0) using the 'hmmpfam' program $[22,23]$.

\section{Genome location of fibrinogen-related proteins}

Perl scripts were used to extract the chromosome location for each gene (or scaffold if chromosome information is not available) and show the distribution of FREPs in the respective genomes of the 12 Drosophila species.

\section{Multiple sequence alignment and phylogenetic analysis} Multiple sequence alignment was performed using the ClustalW for large data set or T_Coffee program $[25,26,35]$. Multiple sequence alignment was visualized using ESPript [36]. For phylogenetic tree construction, the seed sequence was the multiple sequence alignment of FBG domains that excluded truncated FBG domains. Bootstrap was applied to the data. Protein distance was calculated using the Jones-Taylor-Thornton model of change between amino acids and a Hidden Markov Model (HMM) method of inferring different rates of evolution at different amino acid positions. Neighbor-joining was applied to produce the tree. Phylogenetic analysis was carried out with the package from PHYLIP [37], the consensus tree was drawn using the consense program and visualized using the drawgram tool in this package.

\section{Secondary structure prediction}

Secondary structure prediction was produced with the PHD program [27], with multiple sequence alignment of FBG domains. The structure data of TL5A was obtained from protein data bank (PBD) [38].

\section{Microarray data retrieval}

In order to find gene expression profiles in microarray analysis, GEO profiles from NCBI [29] were searched for each member of FREPs from $D$. melanogaster.

\section{Authors' contributions}

SM carried out the database survey. He identified and analyzed the FBG domains, and prepared the manuscript. XW conceived the study and contributed to the preparation of the manuscript. All authors read and approved the final manuscript.

\section{Additional material}

\section{Additional file 1}

The distribution of fibrinogen-like domains in fibrinogen-related proteins. SledgeHMMER was used to carry out batch searching of the current Pfam database (version 20.0) to identify known domains using the 'hmmpfam' program. Gene ID was shown in the left side of each gene. The domains were indicated in the red. The domain name was shown below the domain detected by SledgeHMMER.

Click here for file

[http://www.biomedcentral.com/content/supplementary/14712164-9-260-S1.jpeg]

\section{Additional file 2}

Phylogenetic tree of the fibrinogen-like domains. The seed sequence used for constructing the tree was the multiple sequence alignment of FBG domains that excluded truncated FBG domains. Bootstrap was applied to the data. Protein distance was calculated using the Jones-Taylor-Thornton model of change between amino acids and a Hidden Markov Model (HMM) method of inferring different rates of evolution at different amino acid positions. Neighbor-joining was applied to produce the tree. The FBG domains of each FREP are denoted by their gene name or GLEANR gene ID.

Click here for file

[http://www.biomedcentral.com/content/supplementary/14712164-9-260-S2.pdf] 


\section{Acknowledgements}

We thank Zhao Lai for critical reading and comments. This research was supported in part by the Indiana METACyt Initiative of Indiana University, funded in part through a major grant from the Lilly Endowment, Inc.

\section{References}

I. Gorkun OV, Veklich YI, Weisel JW, Lord ST: The conversion of fibrinogen to fibrin: recombinant fibrinogen typifies plasma fibrinogen. Blood 1997, 89:4407-I4.

2. Gokudan S, Muta T, Tsuda R, Koori K, Kawahara T, Seki N, Mizunoe Y, Wai SN, Iwanaga S, Kawabata S: Horseshoe crab acetyl grouprecognizing lectins involved in innate immunity are structurally related to fibrinogen. Proc Natl Acad Sci USA 1999, 96: 10086-1009|.

3. Wang $X$, Zhao $Q$, Christensen BM: Identification and characterization of the fibrinogen-like domain of fibrinogen-related proteins in the mosquito, Anopheles gambiae, and the fruitfly, Drosophila melanogaster, genomes. BMC Genomics 2005, 6:I 14.

4. Matsushita $M$, Fujita $T$ : The role of ficolins in innate immunity Immunobiology 2002, 205:490-497.

5. Erickson HP: Tenascin-C, tenascin-R and tenascin-X: a family of talented proteins in search of functions. Curr Opin Cell Biol 1993, 5:869-76.

6. Kobayashi R, Mizutani A, Hidaka $\mathrm{H}$ : Isolation and characterization of a 36-kDa microfibril-associated glycoprotein by the newly synthesized isoquinolinesulfonamide affinity chromatography. Biochem Biophys Res Commun I 994, I 98: | 262-6.

7. Endo $Y$, Matsushita M, Fujita T: Role of ficolin in innate immunity and its molecular basis. Immunobiology 2007, 2 I 2:37 I-9.

8. Lu J, Teh C, Kishore U, Reid KB: Collectins and ficolins: sugar pattern recognition molecules of the mammalian innate immune system. Biochim Biophys Acta 2002, 1 572:387-400.

9. Lu J, Le Y: Ficolins and the fibrinogen-like domain. Immunobiology 1998, 199:190-199.

10. Teh C, Le Y, Lee $\mathrm{SH}$, Lu J: M-ficolin is expressed on monocytes and is a lectin binding to $\mathbf{N}$-acetyl-D-glucosamine and mediates monocyte adhesion and phagocytosis of Escherichia coli. Immunology 2000, I 0 I:225-32.

II. Adema CM, Hertel LA, Miller RD, Loker ES: A family of fibrinogen-related proteins that precipitates parasite-derived molecules is produced by an invertebrate after infection. Proc Natl Acad Sci USA 1997, 94:869l-8696.

12. Kenjo A, Takahashi M, Matsushita M, Endo Y, Nakata M, Mizuochi T, Fujita T: Cloning and characterization of novel ficolins from the solitary ascidian, Halocynthia roretzi. J Biol Chem 200I, 276: $19959-19965$

13. Schroder HC, Ushijima H, Krasko A, Gamulin V, Thakur NL, DiehlSeifert B, Muller IM, Muller WE: Emergence and disappearance of an immune molecule, an antimicrobial lectin, in basal metazoa. A tachylectin-related protein in the sponge Suberites domuncula. J Biol Chem 2003, 278:32810-7.

14. Wang X, Rocheleau TA, Fuchs JF, Hillyer JF, Chen CC, Christensen BM: A novel lectin with a fibrinogen-like domain and its potential involvement in the innate immune response of Armigeres subalbatus against bacteria. Insect Mol Biol 2004, I 3:273-82

15. Waterhouse RM, Kriventseva EV, Meister S, Xi Z, Alvarez KS, Bartholomay LC, Barillas-Mury C, Bian G, Blandin S, Christensen BM Dong $Y$, Jiang $H$, Kanost MR, Koutsos AC, Levashina EA, Li J, Ligoxygakis P, Maccallum RM, Mayhew GF, Mendes A, Michel K, Osta MA Paskewitz S, Shin SW, Vlachou D, Wang L, Wei W, Zheng L, Zou Z, Severson DW, Raikhel AS, Kafatos FC, Dimopoulos G, Zdobnov EM, Christophides GK: Evolutionary dynamics of immune-related genes and pathways in disease-vector mosquitoes. Science 2007, 3 I 6(5832): | 738-43.

16. Rubin GM, Yandell MD, Wortman JR, Gabor Miklos GL, Nelson CR Hariharan IK, Fortini ME, Li PW, Apweiler R, Fleischmann W, Cherry JM, Henikoff S, Skupski MP, Misra S, Ashburner M, Birney E, Bogusk MS, Brody T, Brokstein P, Celniker SE, Chervitz SA, Coates D, Cravchik A, Gabrielian A, Galle RF, Gelbart WM, George RA, Goldstein LS, Gong F, Guan P, Harris NL, Hay BA, Hoskins RA, Li J, Li Z, Hynes RO, Jones SJ, KuehI PM, Lemaitre B, Littleton JT, Morrison DK, Mungall C, O'Farrell PH, Pickeral OK, Shue C, Vosshall LB, Zhang J,
Zhao Q, Zheng XH, Lewis S: Comparative genomics of the eukaryotes. Science 2000, 287(546I):2204-I5.

17. Kellis M, Patterson N, Endrizzi M, Birren B, Lander ES: Sequencing and comparison of yeast species to identify genes and regulatory elements. Nature 2003, 423(6937):24I-54.

18. McClelland M, Florea L, Sanderson K, Clifton SW, Parkhill J, Churcher C, Dougan G, Wilson RK, Miller W: Comparison of the Escherichia coli K-I2 genome with sampled genomes of a Klebsiella pneumoniae and three salmonella enterica serovars, Typhimurium, Typhi and Paratyphi. Nucleic Acids Res 2000, 28(24):4974-86.

19. Drosophila 12 Genomes Consortium: Evolution of genes and genomes on the drosophila phylogeny. Nature 2007, 450:203-218.

20. Kairies N, Beisel HG, Fuentes-Prior P, Tsuda R, Muta T, Iwanaga S, Bode W, Huber R, Kawabata S: The 2.0-A crystal structure of tachylectin 5A provides evidence for the common origin of the innate immunity and the blood coagulation systems. Proc Natl Acad Sci USA 200I, 98:13519-24

21. Nozawa M, Nei M: Evolutionary dynamics of olfactory receptor genes in Drosophila species. Proc Natl Acad Sci USA 2007, I 04(I 7):71 22-7.

22. Chukkapalli G, Guda C, Subramaniam S: SledgeHMMER: a web server for batch searching the Pfam database. Nucleic Acids Res 2004:W542-4.

23. SledgeHMMER [http://bioapps.rit.albany.edu/sledgeHMMER/]

24. AAA database [http://rana.lbl.gov/drosophila]

25. Notredame C, Higgins D, Heringa J: T-Coffee: A novel method for multiple sequence alignments. Journal of Molecular Biology 2000, 302:205-217

26. T-Coffee [http://tcoffee.vital-it.ch/cgi-bin/Tcoffee/tcoffee cgi/ index.cgi]

27. PHD program [http://www.predictprotein.org/]

28. Tanio $M$, Kondo $S$, Sugio $S$, Kohno $T$ : Trivalent recognition unit of innate immunity system; crystal structure of trimeric human m-ficolin fibrinogen-like domain. J Biol Chem 2007, 282:3889-3895.

29. GEO profile [http://www.ncbi.nlm.nih.gov/geo/]

30. Vodovar N, Vinals M, Liehl P, Basset A, Degrouard J, Spellman P, Boccard F, Lemaitre B: Drosophila host defense after oral infection by an entomopathogenic Pseudomonas species. Proc Natl Acad Sci USA 2005, I 02(32): I|4|4-9.

31. Johansson KC, Metzendorf C, Soderhall K: Microarray analysis of immune challenged Drosophila hemocytes. Exp Cell Res 2005, 305(I): | 45-55.

32. King-Jones K, Horner MA, Lam G, Thummel CS: The DHR96 nuclear receptor regulates xenobiotic responses in Drosophila. Cell Metab 2006, 4(I):37-48.

33. Flybse [http://flybase.org/]

34. Comparative Annotation Freeze I (CAFI) [http:// insects.eugenes.org/species/data/]

35. Clustalw [http://www.ebi.ac.uk/Tools/clustalw/]

36. Gouet P, Courcelle E, Stuart DI, Metoz F: ESPript: multiple sequence alignments in PostScript. Bioinformatics 1999 , I 5:305-8.

37. Phylip [http://evolution.genetics.washington.edu/phylip.html]

38. Guex N, Peitsch MC: SWISS-MODEL and the Swiss-PdbViewer: an environment for comparative protein modeling. Electrophoresis 1997, I 8:27|4-23. 